\title{
Near-Capacity Irregular Convolutional Coded Cooperative Differential Linear Dispersion Codes Using Multiple-Symbol Differential Decoding Aided Non-coherent Detection
}

\author{
Chao $\mathrm{Xu}$, Soon $\mathrm{Xin} \mathrm{Ng}$ and Lajos Hanzo \\ School of ECS, University of Southampton, SO17 1BJ, United Kingdom. \\ Email: \{cx1g08,sxn,lh\}@ecs.soton.ac.uk, http://www-mobile.ecs.soton.ac.uk
}

\begin{abstract}
We propose a novel near-capacity Multiple-Symbol Differential Decoding (MSDD) aided cooperative Differential Linear Dispersion Code (DLDC), which exhibits a high grade of system design flexibility in terms of the choice of activated relays and the DLDC's rate allocation. More specifically, the system has the freedom to activate a range of DLDCs depending on both the number of relays available in the network, as well as on their position, throughput and complexity considerations.
\end{abstract}

Index Terms-Soft-output Multiple-Symbol Differential Detection, cooperative Differential Linear Dispersion Code, decodeand-forward relaying, code rate allocation.

\section{INTRODUCTION}

Cooperative diversity was proposed in [1], [2], where the single-element Mobile Stations (MSs) share their antennas to form a Virtual Antenna Array (VAA) and as a benefit, they typically experience uncorrelated fading. However, it becomes unrealistic for the relays and the destination to estimate the channel of all the VAA links, especially when the fading fluctuates rapidly. To avoid the potentially excessive complexity of coherent cooperative MIMO detection, noncoherently detected DPSK may be used in each of the singleantenna links. Accordingly, Differential STBCs (DSTBCs) could be found in [3], [4]. Furthermore, a Differential Linear Dispersion Code (DLDC) was proposed in [5]. In the DLDCs, $Q$ modulated symbols are dispersed to $M$ spatial and $T$ temporal dimensions, hence a high design flexibility can be achieved by appropriately configuring $Q, M$ and $T$.

In the absense of channel estimation, Conventional Differential Detection (CDD) generally suffers from a $3 \mathrm{~dB}$ performance penalty, provided that the Doppler frequency is not excessive, while upon increasing the Doppler frequency a pronounced irreducible error floor is formed. Multiple-Symbol Differential Detection (MSDD) was proposed for DPSK in [6] in order to reduce the performance discrepancy. The MSDD observes $N_{w}$ consecutive received symbols and makes a joint decision based on $\left(N_{w}-1\right)$ information symbols. The price paid is that the complexity imposed increases exponentially with $N_{w}$. To mitigate the complexity, Multiple-Symbol Differential Sphere Decoding (MSDSD) was proposed in [7], and a novel MSDSD aided cooperative Amplify-and-Forward (AF)

The financial support of the RC-UK under the auspices of the India-UK Advanced Technology Centre as well as of the China-UK $4^{\text {th }}$ generation wireless systems project and that of the European Union under the auspices of the Optimix project is gratefully acknowledged. design was proposed in [8], where a low BER can only be achieved in the high-SNR region. As a further advance, a soft-output MSDSD designed for DPSK was proposed in [9], while the ML-MSDD was invoked for Differential Space-Time Modulation (DSTM) including DSTBCs and DLDCs in [10]. A hard-output MSDSD for DSTM was proposed in [11].

Against this background, the novel contributions of this paper are: 1) we first propose a soft-output MSDSD for the DSTBC/DLDC scheme, so that MSDSD may be applied for turbo detection in cooperative Decode-and-Forward (DF) systems. 2) We also propose a near-capacity MSDD aided cooperative DLDC scheme, which allows flexible relay selection and cooperative rate allocation.

The following notation is used throughout the paper. A DLDC is described by the nomenclature of $D L D C(M N T Q)$, where $M$ and $N$ indicate the number of transmit and receive antennas, while $T$ and $Q$ denote the number of channel uses and the number of transmitted symbols per block, respectively. Furthermore, $N_{w}$ refers to the MSDD/MSDSD window length.

\section{SYSTEM OVERVIEW}

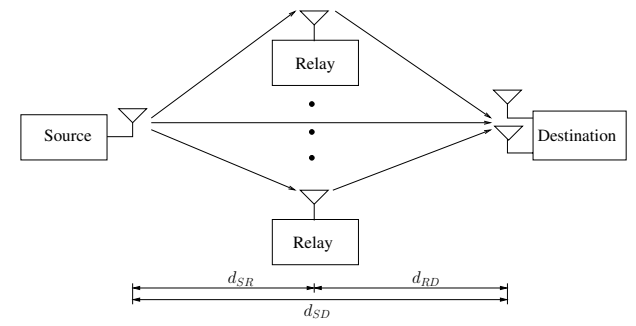

Fig. 1. The block diagram of a relay-aided uplink system.

The schematic of the cooperative system considered is shown in Figure 1, where an Up-Link (UL) scenario is considered. Both the source and the relays are equipped with a single antenna, while the Base Station (BS) has two antennas. As shown in [12], according to free space path loss, the ReducedPathloss-Related (RPLR) power gain of the Source-Relay (SR) link with respect to the Source-Destination (SD) link $G_{S R}$ and the RPLR power gain of the Relay-Destination (RD) link with respect to the SD link $G_{R D}$ have the relationship of:

$$
\frac{1}{\sqrt{G_{S R}}}+\frac{1}{\sqrt{G_{R D}}}=1,
$$

and the terminology of the equivalent transmit SNR at the 


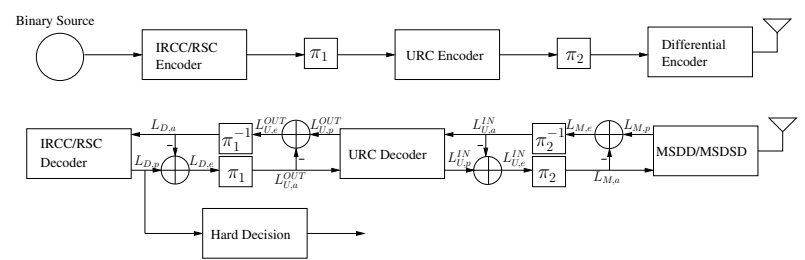

Fig. 2. The schematic of the three-component serial-concatenated encoder at the source and relays, and the corresponding three-stage turbo detection at the relays and the destination.

source was introduced in [12] as ${ }^{1}$ :

$$
\begin{aligned}
\mathrm{SNR}_{\mathrm{t}} & =\mathrm{SNR}_{\mathrm{SR}}-10 \log _{10}\left(G_{S R}\right) \quad \mathrm{dB} \\
& =\mathrm{SNR}_{\mathrm{RD}}-10 \log _{10}\left(G_{R D}\right) \quad \mathrm{dB},
\end{aligned}
$$

where $\mathrm{SNR}_{\mathrm{SR}}$ and $\mathrm{SNR}_{\mathrm{RD}}$ denote the receive SNR for the SR link at the relay as well as for the RD link at the destination.

In order to achieve an infinitesimally low BER, turbo detection may be employed at both the relays and at the destination. Similar to the classic Recursive Systematic Convolutional (RSC) codes, the differential encoder of the DPSK/DSTM has a recursive structure. Hence theoretically a free distance of $d=2$ may be achieved by a combined RSC decoder and a MSDD/MSDSD having a detection window size as long as the encoding frame length. However, the detection window size of MSDSD is severely limited because of its complexity. As an alternative mean, a Unity Rate Code (URC) may be employed as seen in Figure 2. If the relays and the destination are able to afford the IRregular Convolutional Code (IRCC) decoding complexity, then near-capacity performance may be achieved.

In the first transmission period, the source transmits IRCC/RSC coded as well as URC precoded DPSK symbols to both the relays and to the destination. At the relays, hard decisions are made after the three-stage turbo MSDD/MSDSD. Then the IRCC/RSC and URC re-encoded DLDC symbols are transmitted by the relays during the second transmission period. At the destination, the same turbo detection process is carried out for the coded DLDC symbols received from the relays. The overall throughput of the proposed cooperative scheme is given by:

$$
R=\frac{R_{S} \cdot b_{S}}{1+\frac{R_{S} \cdot b_{S}}{R_{R} \cdot\left(Q \cdot b_{R} / T\right)}},
$$

where $R_{S}$ and $R_{R}$ are the code rate of the IRCC/RSC employed at the source and at the relays, respectively. The variables $b_{S}$ and $b_{R}$ denote the bits-per-symbol of the DPSK modulation scheme employed at the source node, and that of the DLDC employed at the relay node.

The choice of the DLDC parameters $M$ and $N$ depends on how many relays are available in the network as well as on how many UL receive antennas are used at the BS. The DLDC throughput specified by $Q \cdot b_{R} / T$ is determined by the position of the relays, which specifies RPLR power gains $G_{S R}$ and $G_{R D}$. Our proposed DLDC selection designed for the cooperative DF scheme is detailed in Section IV.

\footnotetext{
${ }^{1}$ We note that the terminology of $\mathrm{SNR}_{\mathrm{t}}$ has to be treated with extra caution, since it relates the transmit signal power to the noise power, which are - rather unusually - quantities defined at different physical locations.
}

\section{SofT-OUTPUT MSDSD DESIGNED FOR DSTM}

Differential encoding designed for DSTM schemes may be formulated in a way similar to classic DPSK, yielding [13]:

$$
\mathbf{S}_{\mathbf{n}}= \begin{cases}\mathbf{S}_{\mathbf{1}} & n=1 \\ \mathbf{X}_{\mathbf{n}-\mathbf{1}} \mathbf{S}_{\mathbf{n}-\mathbf{1}} & n>1\end{cases}
$$

where the $(T \times T)$-element unitary matrix $\mathbf{X}_{n}$ carries the source information, while the transmission matrix $\mathbf{S}_{n}$ has a size of $(T \times M)$.

To elaborate a little further, normally $M \leqslant T$ is assumed for the transmission matrix $\mathbf{S}_{n}$, because no additional capacity and diversity gain may be attained by further increasing $M$ beyond $T$ [5]. If we have $M=T, \mathbf{S}_{\mathbf{1}}$ in Equation (4) may be represented by any legitimate constellation matrix for DSTBC, or an identity matrix for DLDC. Then all the transmission matrices are unitary. In the case of $M<T, \mathbf{S}_{1}$ should be generated by taking the first $M$ columns of the unitary matrix, and then normalized by a factor of $\sqrt{T / M}$, which is also known as transmit power normalization:

$$
\operatorname{tr}\left(\mathbf{S}_{n} \mathbf{S}_{n}^{H}\right)=T .
$$

We assume that the Rayleigh fading remains constant over $T$ channel uses and that the received signal is contaminated by Additive White Gaussian-distributed Noise (AWGN), hence the received signal may be modelled as:

$$
\mathbf{Y}_{n}=\mathbf{S}_{n} \mathbf{H}_{n}+\mathbf{V}_{n}
$$

where the matrix $\mathbf{Y}_{n}$ has a size of $(T \times N)$. The AWGN matrix $\mathbf{V}_{n}$ has the same size, a zero mean and a variance of $N_{0}$ for each dimension. The channel matrix $\mathbf{H}_{n}$ is of size $(M \times N)$, and it is generated according to Clarke's fading model.

As mentioned before, the MSDD/MSDSD observes $N_{w}$ consecutive received signal blocks $\left\{\mathbf{Y}_{n}\right\}_{n=1}^{N_{w}-1}$ and makes a joint decision based on $\left(N_{w}-1\right)$ consecutive information blocks $\left\{\mathbf{X}_{n}\right\}_{n=1}^{N_{w}-1}$. The hard-output MSDSD designed for the DSTM of [11] should be modified in order to be used in turbo detection. Similarly to the soft-output DPSK MSDSD of [9], the Sphere Decoder (SD) aims to find the optimum $\left(N_{w}-1\right)$ blocks $\left\{\mathbf{X}_{n}\right\}_{n=1}^{N_{w}-1}$ that leads to the minimum Euclidean distance, which is formulated as:

$$
\sum_{i=1}^{N_{w}-1}\left\|\sum_{j=i}^{N_{w}} l_{j i} \mathbf{S}_{\mathbf{j}}^{\mathbf{H}} \mathbf{Y}_{\mathbf{j}}\right\|^{2}-\log \left(\operatorname{Pr}\left(\left\{\mathbf{X}_{\mathbf{n}}\right\}_{n=1}^{N_{w}-1}\right)\right) \leq R^{2}
$$

where $R$ denotes the decoding sphere radius, which is minimized by the SD. The coefficient $l_{j i}$ in Equation (7) represents the predictor coefficients hosted by the corresponding elements in the lower triangular matrix $\mathbf{L}$ in Equation (8) of [9], whose $\mathbf{L}$ is generated from the decomposed channel correlation inversion matrix. Our assumption for DSTM is that the fading is invariant over a DSTM block duration, hence the channel correlation matrix for DSTM MSDSD is the same as that of the DQPSK MSDSD in [9]. The a priori probability in Equation (7) may be calculated by the product of the a priori individual information block probabilities according to the $a$ priori LLRs and the corresponding binary bit combinations. 
The most recent transmission matrix $\mathbf{S}_{N_{w}}$ is a common multiplier for all the transmission matrices. Hence we introduce the accumulated information matrix in order to eliminate the influence of $\mathbf{S}_{N_{w}}$, which may be formulated as:

$$
\mathbf{A}_{n}=\mathbf{S}_{n} \mathbf{S}_{N_{w}}^{H}= \begin{cases}\prod_{i=n}^{N_{w}-1} \mathbf{X}_{i}^{H} & 1 \leqslant n<N_{w} \\ \mathbf{I}_{\mathbf{T}} & n=N_{w} .\end{cases}
$$

Let us now define the Partial Euclidean Distance (PED) component seen in Equation (7) as:

$$
\begin{aligned}
d_{i}^{2}=\sum_{t=i}^{N_{w}-1} & \left(\left\|\sum_{j=t}^{N_{w}} l_{j t} \mathbf{S}_{j}^{H} \mathbf{Y}_{j}\right\|^{2}-\log \left(\operatorname{Pr}\left\{\mathbf{X}_{t}\right\}\right)\right) \\
=d_{i+1}^{2}+ & \left\|l_{i i} \mathbf{X}_{i} \mathbf{Y}_{i}+\mathbf{A}_{i+1}\left(\sum_{j=i+1}^{N_{w}} l_{j i} \mathbf{A}_{j}^{H} \mathbf{Y}_{j}\right)\right\|^{2} \\
& -\log \left(\operatorname{Pr}\left\{\mathbf{X}_{i}\right\}\right),
\end{aligned}
$$

with $i=1,2, \ldots,\left(N_{w}-1\right)$, and it lies within the decoding sphere. Each time the MSDSD performs sphere decoding, the $\left(N_{w}-1\right)$ blocks $\left\{\mathbf{X}_{n}\right\}_{n=1}^{N_{w}-1}$ giving the minimum decoding sphere radius $R$ are found. Therefore the MSDSD constitutes the Max-Log-MAP approximation of the ML-MSDD, where only two optimum combinations are taken into account, which is formulated as:

$$
\log \left(\frac{\operatorname{Pr}\left\{b_{k}=1 \mid \mathbf{Y}\right\}}{\operatorname{Pr}\left\{b_{k}=0 \mid \mathbf{Y}\right\}}\right) \approx d_{M A P}^{b_{k}=1}-d_{M A P}^{b_{k}=0},
$$

where $d_{M A P}^{b_{k}=1}$ and $d_{M A P}^{b_{k}=0}$ denote the minimum Euclidean distance, when $b_{k}$ is fixed to 1 and 0 , respectively. As a result, the reliability of the LLRs is degraded. Therefore, similar to the soft-decision aided MSDSD designed for DPSK, the observation windows are shifted only by one block at a time, while only the LLRs of the central data block are calculated each time, and $N_{w}$ has to be an even number.

The EXtrinsic Information Transfer (EXIT) chart [14] of the soft-output MSDSD decoder designed for DLDC/DSTBC is shown in Figure 3. BPSK signalling was employed and a normalized Doppler frequency of $f_{d}=0.03$ was assumed. Observe in Figure 3 that the performance of DLDC degrades as $Q$ increases, which manifests itself in terms of EXIT curves, which are shifted downward. This is because the average transmit energy assigned to each symbol is decreased. Moreover, also observe in Figure 3 that an increased iteration gain may be achieved, because upon increasing $Q$, more independently faded symbols are detected. The DLDC(3133) and DLDC(3144) schemes have an identical rate and therefore have the same performance. The Differential G3 (DG3) DSTBC scheme [4] has a better performance than the DLDC(3133)/DLDC(3144) arrangement as a benefit of its orthogonal design. Figure 3 also shows that MSDSD aided DSTM codes exhibit an improved iteration gain, as detection window length $N_{w}$ increases. Again, it is expected that the $(1.0,1.0)$ point of perfect convergence may be reached in the EXIT chart, when the detection window length is as long as the frame length, because the process of differential encoding

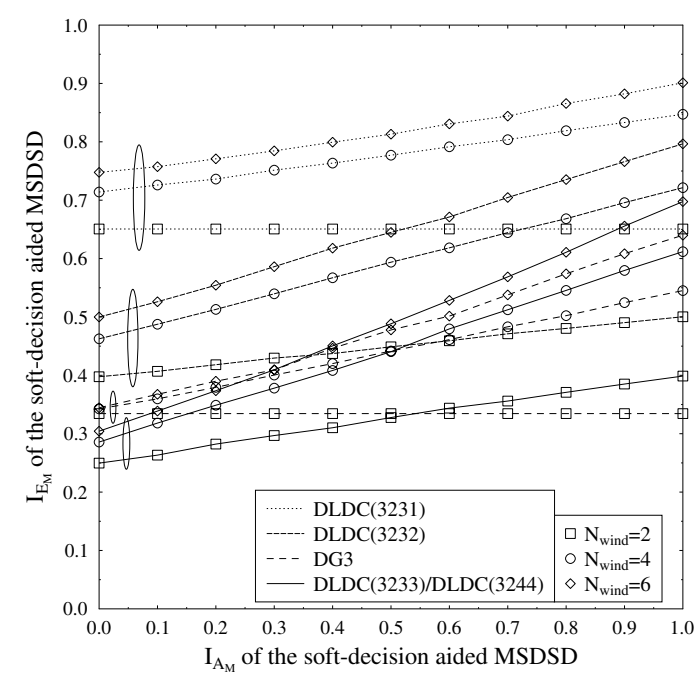

Fig. 3. The EXIT chart of the soft-decision aided MSDSD decoder for $\mathrm{DLDC} / \mathrm{DSTBC}$ at $\mathrm{SNR}=0 \mathrm{~dB}$ and $f_{d}=0.03$.

is recursive. However, the associated exponentially increasing complexity limits the affordable window size in practice.

\section{Design And Performance}

In this section, we propose a specific relay selection and rate allocation design for the near-capacity MSDD aided cooperative DLDC scheme, followed by our simulation results.

Based on EXIT chart estimation and Monte-Carlo simulation $^{2}$, the $\mathrm{SNR}_{\mathrm{RD}}$ required for achieving an infinitesimally low BER at the destination is summarized in Table I. We assume that there are three available relays, hence the class of DLDC(32TQ) and DG3 are of interest. Without loss of generality, we consider the DSTM scheme employing BPSK, i.e. we have $\left(b_{R}=1\right)$, since increasing the number of $Q$ is preferred compared to employing a higher-level modulation scheme. Furthermore, since the diversity gain of DLDC is determined by $N \min \{M, T\}$ [5], a setting of $T=M$ is fixed.

Table I demonstrates that a substantial design flexibility may be provided by the rich set of DSTM schemes. It was demonstrated in [15] that having a flexible code rate allocation was beneficial for the cooperative DF schemes. In this paper, we propose to allocate the code-rate by appropriately choosing the different modulation schemes. The design procedures proposed for our DF relaying scheme are as follows:

1 We first determine the modulation scheme employed at the source based on the throughput requirement and the affordable decoding complexity at the relays, then the $\mathrm{SNR}_{\mathrm{SR}}$ required at the relays for avoiding error propagation is determined.

2 The BS chooses the parameters $M$ and $N$ for DSTM according to the number of available relays, and then sets up the corresponding lookup table. Table I represents the scenario of $M=3$ as well as $N=2$ for $f_{d}=0.03$.

\footnotetext{
${ }^{2}$ It has been widely exploited [13] that a vanishingly low BER is achievable, when an open EXIT chart tunnel is formed between the EXIT curves of the inner and outer code. However, in practice not all received frames can be error-freely decoded at the EXIT chart estimated SNR, since the EXIT chart is only accurate for an infinite interleaver length. In practical Monte-Carlo simulations, normally about $0.5 \mathrm{~dB}$ higher SNR is required.
} 
TABLE I

LOOKUP TABLE SUMMARIZING THE SNR RD REQUIRED FOR ACHIEVING AN INFINITESIMALLY LOW BER FOR THE THREE-STAGE TURBO DETECTED IRCC-URC-DLDC(323Q)/DG3 MSDD SCHEME, WHEN $f_{d}=0.03$

\begin{tabular}{|c|c|c|c|}
\hline $\begin{array}{l}\text { URC- } \\
\text { DLDC(3231) }\end{array}$ & $\begin{array}{ll}\mathrm{SNR}_{\mathrm{RD}} & \text { for } \\
N_{w}=2 & \\
\end{array}$ & $\begin{array}{ll}\mathrm{SNR}_{\mathrm{RD}} & \text { for } \\
N_{w}=4 & \\
\end{array}$ & $\begin{array}{ll}\mathrm{SNR}_{\mathrm{RD}} & \text { for } \\
N_{w}=6 & \\
\end{array}$ \\
\hline 1 inner iteration & $-3.6 \mathrm{~dB}$ & $-4.4 \mathrm{~dB}$ & $-4.7 \mathrm{~dB}$ \\
\hline 2 inner iterations & $-3.6 \mathrm{~dB}$ & $-5.2 \mathrm{~dB}$ & $-5.8 \mathrm{~dB}$ \\
\hline $\begin{array}{l}\text { URC- } \\
\text { DLDC(3232) }\end{array}$ & $\begin{array}{ll}\mathrm{SNR}_{\mathrm{RD}} & \text { for } \\
N_{w}=2 & \end{array}$ & $\begin{array}{ll}\mathrm{SNR}_{\mathrm{RD}} & \text { for } \\
N_{w}=4 & \end{array}$ & $\begin{array}{ll}\mathrm{SNR}_{\mathrm{RD}} & \text { for } \\
N_{w}=6 & \end{array}$ \\
\hline 1 inner iteration & $-1.2 \mathrm{~dB}$ & $-2.1 \mathrm{~dB}$ & $-2.4 \mathrm{~dB}$ \\
\hline 2 inner iterations & $-1.6 \mathrm{~dB}$ & $-3.2 \mathrm{~dB}$ & $-3.7 \mathrm{~dB}$ \\
\hline $\begin{array}{l}\text { URC- } \\
\text { DLDC(3233) }\end{array}$ & $\begin{array}{ll}\mathrm{SNR}_{\mathrm{RD}} & \text { for } \\
N_{w}=2 & \\
\end{array}$ & $\begin{array}{ll}\mathrm{SNR}_{\mathrm{RD}} & \text { for } \\
N_{w}=4 & \\
\end{array}$ & $\begin{array}{l}\mathrm{SNR}_{\mathrm{RD}} \\
N_{w}=6\end{array}$ \\
\hline 1 inner iteration & $0.4 \mathrm{~dB}$ & $-0.5 \mathrm{~dB}$ & $-0.8 \mathrm{~dB}$ \\
\hline 2 inner iterations & $-0.2 \mathrm{~dB}$ & $-1.7 \mathrm{~dB}$ & $-2.2 \mathrm{~dB}$ \\
\hline $\begin{array}{ll}\text { URC-DG3 with } \\
\text { 2Rxs }\end{array}$ & $\begin{array}{ll}\mathrm{SNR}_{\mathrm{RD}} & \text { for } \\
N_{w}=2 & \\
\end{array}$ & $\begin{array}{ll}\mathrm{SNR}_{\mathrm{RD}} & \text { for } \\
N_{w}=4 & \\
\end{array}$ & $\begin{array}{l}\mathrm{SNR}_{\mathrm{RD}} \\
N_{w}=6\end{array}$ \\
\hline 1 inner iteration & $-0.1 \mathrm{~dB}$ & $-0.7 \mathrm{~dB}$ & $-0.9 \mathrm{~dB}$ \\
\hline 2 inner iterations & $-0.1 \mathrm{~dB}$ & $-1.7 \mathrm{~dB}$ & $-2.3 \mathrm{~dB}$ \\
\hline
\end{tabular}

3 The RPLR power gains $G_{S R}$ and $G_{R D}$ are determined by the positions of the activated relays. Then the $\mathrm{SNR}_{\mathrm{RD}}$ required at the destination may be calculated by $\mathrm{SNR}_{\mathrm{RD}}=\mathrm{SNR}_{\mathrm{SR}}-10 \log _{10}\left(G_{S R}\right)+10 \log _{10}\left(G_{R D}\right)$.

4 Finally, the BS find a suitable code rate for the DLDC in the lookup table using the estimated $\mathrm{SNR}_{\mathrm{RD}}$.

It can be seen that the closer the relays approach the source node, the higher $G_{S R}$ becomes compared to $G_{R D}$, which leads to a lower $\mathrm{SNR}_{\mathrm{RD}}$ required at the destination. As a result, according to Table I, a lower DLDC code-rate has to be chosen for the weak RD link. By contrast, a higher-rate DLDC should be selected in the opposite situation.

We now present a design example. The modulation scheme of the SR link is first fixed to be DQPSK, and the MSDD having a window length of $N_{w}=4$ is employed at the relays. The EXIT chart and decoding trajectory recorded for the SR links are shown in Figure 4, where our 36component IRCC [16] has the weighting coefficients of $\left[\alpha_{1}, \cdots, \alpha_{36}\right]=[0,0,0,0,0,0.29678,0.284534,0.0698805$, $0,0,0.0470204,0.161304,0,0,0.0864003,0,0,0,0,0,0,0,0$, $0,0,0,0,0,0,0,0,0,0,0,0,0.0541135]$. The bit interleaver length is set to $10^{6}$. It can be seen in Figure 4 that $\mathrm{SNR}_{\mathrm{SR}}=5.7 \mathrm{~dB}$ is required for achieving a vanishingly low $\mathrm{BER}$ at the relays. If the relays cannot afford the complexity of decoding the 36-component IRCC and/or using MSDD, then a single-component RSC as well as the MSDSD designed in Section III may be employed, and the bit interleaver length may be reduced to $10^{4}$. It can be seen in Figure 4 that employing a single-component RSC and MSDSD requires a $0.8 \mathrm{~dB}$ higher SNR at the relays.

As an example, we assume that there are three available relays, which are located in a same SNR region, where $G_{S R}$ is $9 \mathrm{~dB}$ higher than $G_{R D}$. According to the RPLR power gains relationship of Equation (1), we have $G_{S R} \approx 11.63 \mathrm{~dB}$ and $G_{R D} \approx 2.63 \mathrm{~dB}$, which requires $\mathrm{SNR}_{\mathrm{RD}}=-3.3 \mathrm{~dB}$ at the destination node. Therefore, based on row $x$ and column $y$ of Table I, the DLDC(3232) scheme using $N_{w}=4$ for MSDD and two inner iterations within the URC-MSDD composite decoder may be selected, as indicated by the bold entry in Table I. As a result, according to Equation (3), the overall

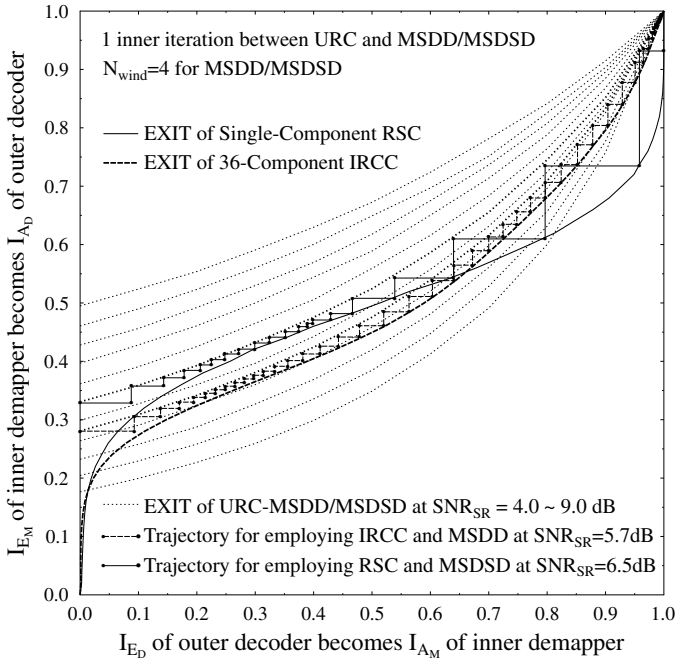

Fig. 4. EXIT chart of the three-stage turbo detected DQPSK scheme of Figure 2 for the SR links, when $f_{d}=0.03$. The 36-component IRCC having weighting coefficients $\left[\alpha_{1}, \cdots, \alpha_{36}\right]=[0,0,0,0,0,0.29678,0.284534$, $0.0698805,0,0,0.0470204,0.161304,0,0,0.0864003,0,0,0,0,0,0,0,0$, $0,0,0,0,0,0,0,0,0,0,0,0,0.0541135]$ and MSDD with $N_{w}=4$ are adopted, while a single inner iterations within the URC-MSDD composite decoder and a interleaver length of $10^{6}$ are employed. The case of employing RSC and MSDSD with a interleaver length of $10^{4}$ is also drawn.

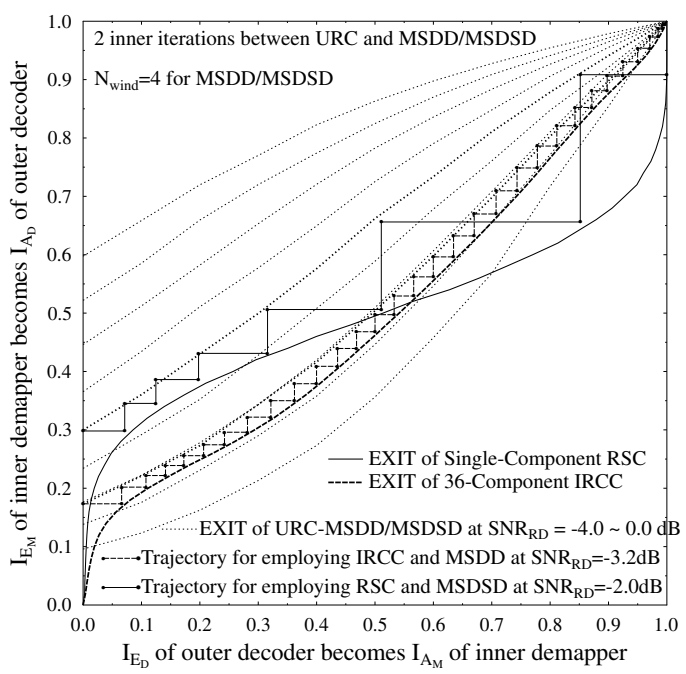

Fig. 5. EXIT chart of the three-stage turbo detected DLDC(3232) scheme of Figure 2 for the RD links, when $f_{d}=0.03$. The 36-component IRCC having weighting coefficients $\left[\alpha_{1}, \cdots, \alpha_{36}\right]=[0,0,0,0.310327,0,0$ $0.113319,0,0.133812,0,0,0.149363,0,0,0.0922107,0,0.070601,0,0,0$, $0,0,0.0755869,0,0,0,0,0,0,0,0,0,0,0,0,0.0548512]$ and the MSDD with $N_{w}=4$ are adopted, while two inner iterations within the URC-MSDD composite decoder and a interleaver length of $10^{6}$ are employed. The case of employing RSC and MSDSD with a interleaver length of $10^{4}$ is also drawn.

throughput of the cooperative scheme is 0.25 . The EXIT chart and decoding trajectory recorded for the scheme selected for the RD links is shown in Figure 5, where the IRCC employed has the weighting coefficients of $\left[\alpha_{1}, \cdots, \alpha_{36}\right]=$ $[0,0,0,0.310327,0,0,0.113319,0,0.133812,0,0,0.149363,0$, $0,0.0922107,0,0.070601,0,0,0,0,0,0.0755869,0,0,0,0,0,0$, $0,0,0,0,0,0,0.0548512]$.

The BER performance evaluated at the destination is portrayed in Figure 6. The maximum achievable rates indicated 


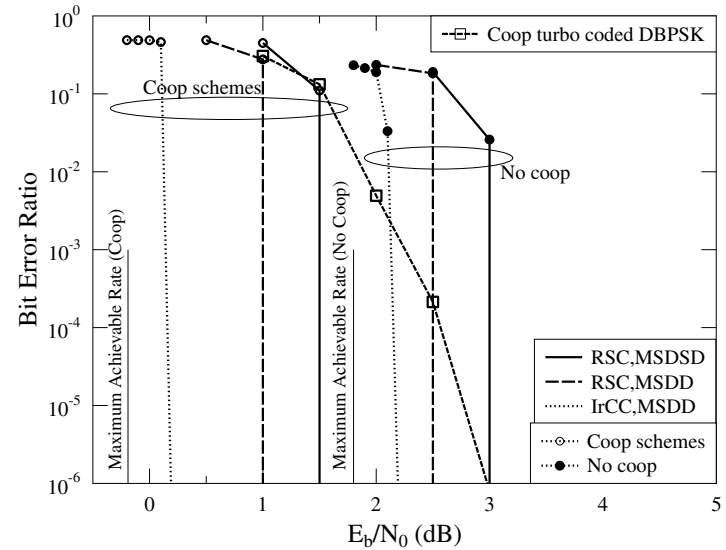

Fig. 6. BER performance of the proposed cooperative DLDC employing IRCC/RSC and MSDD/MSDSD, in comparison with their non-cooperative counterparts, for $f_{d}=0.03$. A MSDSD aided cooperative turbo-coded DBPSK scheme is also drawn as a benchmark.

in Figure 6 are calculated based on the EXIT chart of Figure 4 and 5. More explicitly, it was shown in [14] that the area under the EXIT curves corresponds to the achievable rate. Since half-rate channel codes are employed in our system, the SNR required for the EXIT curves to exhibit a normalized area of $\frac{1}{2}$ is estimated. It is shown in Figure 6 that as a benefit of the cooperative diversity attained, the proposed nearcapacity cooperative scheme provides a $2.0 \mathrm{~dB}$ performance improvement compared to the conventional direct transmission operating without relaying in the specific scenario considered. Furthermore, the low complexity cooperative scheme employing the single-component RSC as well as MSDSD also outperforms its non-cooperative counterpart by $1.5 \mathrm{~dB}$. Figure 6 shows that the performance of employing MSDD is better than that of employing MSDSD, because the MSDSD implements the max-log-approximation of ML MSDD, as demonstrated in Section III.

As another benchmark, a low complexity MSDSD aided cooperative turbo-coded DBPSK scheme with the same system throughput of 0.25 and the same MSDSD window length of $N_{w}=4$ is also portrayed in Figure 6. For the schematics of the cooperative turbo-coded DBPSK, the blocks of IRCC/RSC and URC are replaced by a single half-rate turbo code [17] in the schematic of the serial concatenated code of Figure 2. The number of inner iterations within the turbo decoder is set to 2 , and the number of outer iterations between the turbo decoder and the MSDSD is also set to 2. Figure 6 shows that all the proposed cooperative schemes outperform the benchmark. Observe furthermore that the non-cooperative RSC coded and URC precoded DQPSK's performance is comparable to the cooperative turbo-coded DBPSK. This is because the threestage turbo detection employed in our proposed scheme has a clear open tunnel leading to the $(1.0,1.0)$ point of the EXIT chart, as shown in Figures 4 and 5, which results in a sharp turbo-cliff when decoding convergence takes place.

\section{CONCLusions}

In this paper, we have derived a novel soft-decision aided MSDSD designed for DSTM in the spirit of the soft-output
DPSK MSDSD of [9] and the hard-decision aided DSTM MSDSD of [11]. Furthermore, we have proposed a nearcapacity MSDD aided cooperative DLDC scheme, relying on a flexible relay selection and rate allocation design. We have demonstrated that near-capacity performance may be achieved by the employment of our 36-component IRCC, and the proposed cooperative scheme outperforms both the conventional direct transmission regime operating without relaying and its cooperative turbo-coded DBPSK counterpart.

\section{REFERENCES}

[1] J. Laneman and G. Wornell, "Distributed space-time-coded protocols for exploiting cooperative diversity in wireless networks," IEEE Transactions on Information Theory, vol. 49, pp. 2415-2425, Oct. 2003.

[2] J. Laneman, D. Tse, and G. Wornell, "Cooperative diversity in wireless networks: Efficient protocols and outage behavior," IEEE Transactions on Information Theory, vol. 50, pp. 3062-3080, Dec. 2004.

[3] V. Tarokh and H. Jafarkhani, "A differential detection scheme for transmit diversity," IEEE Journal on Selected Areas in Communications, vol. 18, pp. 1169-1174, July 2000.

[4] H. Jafarkhani and V. Tarokh, "Multiple transmit antenna differential detection from generalized orthogonal designs," IEEE Transactions on Information Theory, vol. 47, pp. 2626-2631, Sept. 2001.

[5] B. Hassibi and B. Hochwald, "Cayley differential unitary space-time codes," IEEE Transactions on Information Theory, vol. 48, pp. 14851503, June 2002.

[6] P. Ho and D. Fung, "Error performance of multiple-symbol differential detection of PSK signals transmitted over correlated rayleigh fading channels," IEEE Transactions on Communications, vol. 40, pp. 15661569, Oct. 1992.

[7] L. Lampe, R. Schober, V. Pauli, and C. Windpassinger, "Multiple-symbol differential sphere decoding," IEEE Transactions on Communications, vol. 53, pp. 1981-1985, Dec. 2005.

[8] L. Wang and L. Hanzo, "The amplify-and-forward cooperative uplink using multiple-symbol differential sphere-detection," Signal Processing Letters, IEEE, vol. 16, pp. 913 -916, Oct. 2009.

[9] V. Pauli, L. Lampe, and R. Schober, “"Turbo DPSK" using soft multiplesymbol differential sphere decoding," IEEE Transactions on Information Theory, vol. 52, pp. 1385-1398, Apr. 2006.

[10] R. Schober and L.-J. Lampe, "Noncoherent receivers for differential space-time modulation," IEEE Transactions on Communications, vol. 50, pp. 768-777, May 2002.

[11] V. Pauli and L. Lampe, "Tree-search multiple-symbol differential decoding for unitary space-time modulation," IEEE Transactions on Communications, vol. 55, pp. $1567-1576$, Aug. 2007.

[12] H. Ochiai, P. Mitran, and V. Tarokh, "Design and analysis of collaborative diversity protocols for wireless sensor networks," in IEEE 60th Vehicular Technology Conference, 2004. VTC2004-Fall. 2004, vol. 7, pp. 4645-4649, Sept. 2004.

[13] L. Hanzo, O. Alamri, M. El-Hajjar, and N. Wu, Near-Capacity MultiFunctional MIMO Systems - Sphere-Packing, Iterative Detection and Cooperation. Wiley-IEEE Press, 2009.

[14] M. Tuchler, "Design of serially concatenated systems depending on the block length," IEEE Transactions on Communications, vol. 52, pp. 209 - 218, Feb. 2004.

[15] L. Wang and L. Hanzo, "Optimum time resource allocation for TDMAbased differential decode-and-forward cooperative systems: a capacity perspective," IEEE Communications Letters, vol. 14, pp. 506 -508, June 2010.

[16] L. Kong, S. Ng, R. Tee, R. Maunder, and L. Hanzo, "Reducedcomplexity near-capacity downlink iteratively decoded generalized multi-layer space-time coding using irregular convolutional codes," IEEE Transactions on Wireless Communications, vol. 9, pp. $684-695$, Feb. 2010.

[17] C. Berrou and A. Glavieux, "Near optimum error correcting coding and decoding: turbo-codes," IEEE Transactions on Communications, vol. 44 pp. $1261-1271$, Oct. 1996. 